\title{
A Time-Oscillating Hartree-Type Schrödinger Equation
}

\author{
Xu Chen \\ Department of Mathematics, Zhejiang University, Hangzhou 310027, China
}

Correspondence should be addressed to Xu Chen; hanzh_0102@163.com

Received 14 March 2014; Revised 19 May 2014; Accepted 19 May 2014; Published 9 June 2014

Academic Editor: Claudio Cuevas

Copyright (C) $2014 \mathrm{Xu}$ Chen. This is an open access article distributed under the Creative Commons Attribution License, which permits unrestricted use, distribution, and reproduction in any medium, provided the original work is properly cited.

We consider the time-oscillating Hartree-type Schrödinger equation $i u_{t}+\Delta u+\theta(\omega t)\left(|x|^{-\gamma} *|u|^{2}\right) u=0$, where $\theta$ is a periodic function. For the mean value $I(\theta)$ of $\theta$, we show that the solution $u_{\omega}$ converges to the solution of $i U_{t}+\Delta U+I(\theta)\left(|x|^{-\gamma} *|U|^{2}\right) U=0$ for their local well-posedness and global well-posedness.

\section{Introduction}

In this paper, we discuss the following Hartree-type Schrödinger equation:

$$
\begin{gathered}
i u_{t}+\Delta u+\theta(\omega t)\left(|x|^{-\gamma} *|u|^{2}\right) u=0, \\
u(0)=\varphi \in H^{1}\left(\mathbb{R}^{N}\right),
\end{gathered}
$$

where $*$ represents the convolution operator, $\gamma \in(0,4] \cap$ $(0, N), \omega \in \mathbb{R}$, and $\theta$ is a periodic function belonging to $C^{1}(\mathbb{R}, \mathbb{R})$. People are interested in Hartree equation since it has many applications in the quantum theory of large systems of nonrelativistic bosonic atoms and molecules. The numbers of bosons in such systems are very large, but the interactions between them are weak. Hartree equation arises in the study of the mean-field limit of such systems; see, for example, [1$3]$.

Different from the classical Hartree-type Schrödinger equation, the coefficient of nonlinearity of (OHS) is a function, especially a periodic function, not some constant, although its $L^{\infty}$ norm is finite. We assume $\tau$ is the period of $\theta$; then we can define the mean value

$$
I(\theta)=\frac{1}{\tau} \int_{0}^{\tau} \theta(s) d s
$$

One can take such mean value as the coefficient of nonlinearity of Hartree-type Schrödinger equation:

$$
\begin{gathered}
i U_{t}+\Delta U+I(\theta)\left(|x|^{-\gamma} *|U|^{2}\right) U=0, \\
U(0)=\varphi \in H^{1}\left(\mathbb{R}^{N}\right) .
\end{gathered}
$$

Then, (OHS) is a time-oscillating equation and (HS) is the corresponding deterministic one. In this paper, our purpose is to discuss the relationship of well-posedness of solutions between (OHS) and (HS).

The Cauchy problem has been settled by Cazenave and Weissler $[4,5]$ and Miao et al. [6-8]. For the sake of conciseness, we only state the results without any detailed proof. The definition of admissible pair is arranged in Section 2, although we use it here.

Proposition 1. For any initial data $\varphi \in H^{1}\left(\mathbb{R}^{N}\right)$, there exists a unique $H^{1}$ solution of $(\mathrm{OHS})$ (or $\left.(H S)\right)$ defined on the maximal life interval $\left(-T_{\min }, T_{\max }\right)$ with $0<T_{\max }, T_{\min } \leq \infty$. Moreover, the following properties hold.

(1) $u \in C\left(\left(-T_{\min }, T_{\max }\right), H^{1}\left(\mathbb{R}^{N}\right)\right) \cap L_{\mathrm{loc}}^{q}\left(\left(-T_{\min }, T_{\max }\right)\right.$, $\left.W^{1, r}\left(\mathbb{R}^{N}\right)\right)$ for any admissible pair $(q, r)$;

(2) (blow-up alternative) if $T_{\max }<\infty$ (resp., $T_{\min }<\infty$ ), then, for $\gamma<4$, one has $\lim _{t \uparrow T_{\max }}\|\nabla u\|_{L^{2}\left(\mathbb{R}^{N}\right)}=+\infty$ and, for $\gamma=4$, one has $\|u\|_{L^{3}\left(\left(0, T_{\max }\right), L^{6 N /(3 N-10)}\left(\mathbb{R}^{N}\right)\right)}=$ $+\infty$ 
As mentioned above, we are concerned with the behavior of solution of (OHS), when $|\omega| \rightarrow+\infty$. Precisely, in the maximal life interval of solution of (HS), we attempt to find the relationship of solutions between (OHS) and (HS) as $|\omega|$ is sufficiently large. Mimicking the approach of Cazenave and Scialom [9] and Fang and Han [10] in the case of the $H^{1}$ Schrödinger equation with the local nonlinear term, we obtain the following theorems for Hartree-type.

Theorem 2. Assume the initial data $\varphi \in H^{1}\left(\mathbb{R}^{N}\right)$ and define $u_{\omega}$ as the solutions of $(\mathrm{OHS})$. Let $U$ be the solution of $(H S)$ with the maximal life interval $\left[0, S_{\max }\right)$. Then, we have

(1) for any time $T$ satisfying $0<T<S_{\max }$, if $|\omega|$ is sufficiently large, the solution $u_{\omega}$ of $(\mathrm{OHS})$ exists in $[0, T]$;

(2) for any admissible pair $(q, r)$ and time $0<T<S_{\max }$, $u_{\omega} \rightarrow U$ in $L^{q}\left((0, T), W^{1, r}\left(\mathbb{R}^{N}\right)\right)$ as $|\omega| \rightarrow \infty$. In particular, the convergence holds in $C\left([0, T], H^{1}\left(\mathbb{R}^{N}\right)\right)$.

Theorem 2 describes the relationship of local wellposedness of solutions between (OHS) and (HS). Furthermore, if the solution of (HS) is globally existent, that is, $S_{\max }=$ $+\infty$, we want to know whether Theorem 2 still holds. The following theorem gives the positive answer if the solution of (HS) owns sufficient decay as $t \rightarrow \infty$.

Theorem 3. Under the assumptions of Theorem 2, suppose that $\gamma>2$ and

$$
U \in L^{12 /(8-\gamma)}\left(\left(0, S_{\max }\right), L^{6 N /(3 N-2-2 \gamma)}\left(\mathbb{R}^{N}\right)\right) ;
$$

and then it follows that solution $U$ of $(H S)$ is global; that is, $S_{\max }=\infty$. Moreover, solution $u_{\omega}$ of $(O H S)$ is also global if $|\omega|$ is sufficiently large, and $u_{\omega} \rightarrow U$ in $L^{q}\left((0, \infty), W^{1, r}\left(\mathbb{R}^{N}\right)\right)$ as $|\omega| \rightarrow \infty$, for all admissible pairs $(q, r)$.

The assumption (2) makes sure the solution $U$ of (HS) owning sufficient decay, by which deduces $U$ not only is global but also has scattering state (the details can be referred to in $[6-8])$. In fact, (2) shows that $U$ is global when $\gamma=4$ immediately, according to the blow-up alternative in Proposition 1. And for $\gamma<4$, the norm of $\|\nabla U(t)\|_{L^{2}\left(\mathbb{R}^{N}\right)}$ can be controlled by (2), for any $t \in\left[0, S_{\max }\right)$, which shows $S_{\max }=\infty$ by the blow-up alternative in Proposition 1. The details can be found in Lemma 9.

Many people show that the condition (2) holds in different cases. Cazenave in [4] shows (2) is true for defocusing case $(I(\theta))$ when $2<\gamma<4$. When $\gamma=4$, Miao et al. in [6] show (2) is true for defocusing case with the radial initial data and for focusing case with the radial initial data and its energy and kinetic energy smaller than the ground state's.

When solution $U$ of (HS) is global but (2) does not hold, we are not sure the behavior of solution $u_{\omega}$ of (OHS) even $\omega$ is sufficiently large. In order to have a good understanding of the development of $u_{\omega}$, we think that we should understand the development of $U$ firstly, especially the blow-up rate of $U$.

In Section 2, we introduce some notations and some useful lemmas. Theorem 2 is proved in Section 3, and Section 4 is devoted to proving Theorem 3.

\section{Notations and Some Tools}

In this section, we introduce some notations and useful lemmas. In order to discuss nonlinear Schrödinger equation conveniently, we always consider the equivalence of (OHS) (or (HS)):

$$
\begin{aligned}
u(t)=e^{i t \Delta} \varphi+i \int_{0}^{t} e^{i(t-s) \Delta} \theta(\omega s) & \\
& \times\left(|x|^{-\gamma} *|u|^{2}\right) u(s) d s
\end{aligned}
$$

where $\left(e^{i t \Delta}\right)_{t \in \mathbb{R}}$ represents the Schrödinger group.

Definition 4 (admissible pair). A pair $(q, r)$ is called admissible if $(2 / q)+(n / r)=n / 2$ and $2 \leq q, r \leq \infty$ (if $N=1$, then $2 \leq r \leq \infty$; if $N=2$, then $2 \leq r<\infty)$.

Before stating the useful lemma, we describe the Classical Strichartz estimates. The proofs of Strichartz estimates are referred to in $[5,11-14]$.

Lemma 5 (classical Strichartz estimates). The following properties hold.

(i) For any $\varphi \in L^{2}\left(\mathbb{R}^{N}\right)$ and any admissible pair $(q, r)$, the function $t \mapsto e^{i t \Delta} \varphi$ belongs to

$$
L^{q}\left(\mathbb{R}, L^{r}\left(\mathbb{R}^{N}\right)\right) \cap C\left(\mathbb{R}, L^{2}\left(\mathbb{R}^{N}\right)\right) .
$$

In addition, there exists a constant $C$ such that

$$
\left\|e^{i t \Delta} \varphi\right\|_{L^{q}\left(\mathbb{R}, L^{r}\right)} \leq C\|\varphi\|_{L^{2}} .
$$

(ii) Let I be an interval in $\mathbb{R}, J=\bar{I}$, and $t_{0} \in J$. If $(\gamma, \rho)$ is an admissible pair and $f \in L^{\gamma^{\prime}}\left(I, L^{\rho^{\prime}}\left(\mathbb{R}^{N}\right)\right)$, then for any admissible pair $(q, r)$, the function

$$
f \longmapsto \int_{t_{0}}^{t} e^{i(t-s) \Delta} f(s) d s, \quad \text { where } t \in I,
$$

belongs to $L^{q}\left(\mathbb{R}, L^{r}\left(\mathbb{R}^{N}\right)\right) \cap C\left(\mathbb{R}, L^{2}\left(\mathbb{R}^{N}\right)\right)$. Moreover, there exists a constant $C$ independent of I such that

$$
\left\|\int_{t_{0}}^{t} e^{i(t-s) \Delta} f(s) d s\right\|_{L^{q}\left(I, L^{r}\right)} \leq C\|f\|_{L^{\gamma^{\prime}\left(I, L^{\rho^{\prime}}\right)}} .
$$

We also need the following maximal estimate, which follows immediately from the sharp Hardy inequality (see [15]).

Lemma 6. Let $0<\gamma<N$; one has

$$
\left\||x|^{-\gamma} *|u|^{2}\right\|_{L^{\infty}\left(\mathbb{R}^{N}\right)} \leqslant\|u\|_{\dot{H}^{\gamma / 2}\left(\mathbb{R}^{N}\right)}^{2} .
$$

The following lemma is the key to discussing the relationship between (OHS) and (HS), which shows that when $|\omega|$ goes to infinity, the nonlinearity of (OHS) converges to the nonlinearity of (HS). The lemma has been proved by Cazenave and Scialom [9]; therefore, we only state it here without any detailed proof. 
Lemma 7. Let $(\gamma, \rho)$ be an admissible pair, and fix a time $t_{0}$. Given $f \in L^{\gamma^{\prime}}\left(\mathbb{R}, L^{\rho^{\prime}}\left(\mathbb{R}^{N}\right)\right)$, it follows that

$$
\int_{t_{0}}^{t} \theta(\omega s) e^{i(t-s) \Delta} f(s) d s \underset{|\omega| \rightarrow \infty}{\longrightarrow} I(\theta) \int_{t_{0}}^{t} e^{i(t-s) \Delta} f(s) d s,
$$

in $L^{q}\left(\mathbb{R}, L^{r}\left(\mathbb{R}^{N}\right)\right)$, for any admissible pair $(q, r)$.

Lemma 8. Let the initial data $\varphi \in H^{1}\left(\mathbb{R}^{N}\right)$. For any $\omega \in \mathbb{R}$, define $u_{\omega}$ as the solution of (OHS), and $U$ is the solution of $(H S)$ with the maximal life interval $\left[0, S_{\max }\right)$. Fix a time l satisfying $0<l<S_{\max }$, and suppose $u_{\omega}$ exists in the interval $[0, l]$ when $|\omega|$ is sufficiently large. Suppose the following conditions hold:

$$
\limsup _{|\omega| \rightarrow \infty}\left\|u_{\omega}\right\|_{X(0, l)}<\infty
$$

where

$$
X(0, l)=\left\{\begin{array}{c}
L^{\infty}\left((0, l), H^{1}\left(\mathbb{R}^{N}\right)\right), \quad \text { if } \gamma \leq 2 ; \\
L^{6 /(\gamma-2)}\left((0, l), L^{6 N /(3 N-2-2 \gamma)}\left(\mathbb{R}^{N}\right)\right) \\
\cap L^{\infty}\left((0, l), H^{1}\left(\mathbb{R}^{N}\right)\right), \quad \text { if } 2<\gamma \leq 4 .
\end{array}\right.
$$

Then, for any admissible pair $(q, r)$, one has

$$
\left\|u_{\omega}-U\right\|_{L^{q}\left((0, l), W^{1, r}\left(\mathbb{R}^{N}\right)\right)} \underset{|\omega| \rightarrow \infty}{\longrightarrow} 0 .
$$

Proof. From the conditions (10), we can choose two constants $L$ and $M$ such that when $|\omega| \geq L$, we have

$$
\sup _{|\omega| \geq L}\left\|u_{\omega}\right\|_{X(0, l)} \leq M
$$

Set

$$
Q=\|U\|_{X(0, l)},
$$

and then Proposition 1 deduces $Q<\infty$.

It follows from (3) that $u_{\omega}-U=i\left(I_{1}+I_{2}\right)$, where

$$
\begin{gathered}
I_{1}=\int_{0}^{t} \theta(\omega s) e^{i(t-s) \Delta}\left[\left(|x|^{-\gamma} *\left|u_{\omega}\right|^{2}\right) u_{\omega}(s)\right. \\
\left.-\left(|x|^{-\gamma} *|U|^{2}\right) U(s)\right] d s, \\
I_{2}=\int_{0}^{t}[\theta(\omega s)-I(\theta)] e^{i(t-s) \Delta}\left(|x|^{-\gamma} *|U|^{2}\right) U(s) d s .
\end{gathered}
$$

By Lemma 6, Hardy-Littlewood-Sobolev inequality, Hölder inequality, and Sobolev embedding, we obtain

$$
\left\|\left(|x|^{-\gamma} *|U|^{2}\right) U\right\|_{Y(0, l)} \leqslant l^{\alpha}\|U\|_{X(0, l)}^{3} \leqslant l^{\alpha} Q^{3},
$$

where

$$
\begin{gathered}
Y(0, l)=\left\{\begin{aligned}
L^{1}\left((0, l), H^{1}\left(\mathbb{R}^{N}\right)\right), & \text { if } \gamma \leq 2 ; \\
L^{6 /(8-\gamma)}\left((0, l), W^{1,6 N /(3 N+2 \gamma-4)}\left(\mathbb{R}^{N}\right)\right), & \text { if } 2<\gamma \leq 4,
\end{aligned}\right. \\
\alpha= \begin{cases}1, & \text { if } \gamma \leq 2 ; \\
2-\frac{\gamma}{2}, & \text { if } 2<\gamma \leq 4 .\end{cases}
\end{gathered}
$$

Therefore, we can obtain from Strichartz estimates and Lemma 7 that

$$
\left\|I_{2}\right\|_{L^{q}\left((0, l) W^{1, r}\left(\mathbb{R}^{N}\right)\right)}:=C \varepsilon_{\omega} \underset{|\omega| \rightarrow \infty}{\longrightarrow} 0 .
$$

It follows from Strichartz estimates, Lemma 6, HardyLittlewood-Sobolev inequality, Hölder inequality, and Sobolev embedding that

$$
\begin{gathered}
\left\|I_{1}\right\|_{L^{q}\left((0, l) W^{1, r}\left(\mathbb{R}^{N}\right)\right)} \\
\leq C l^{\alpha}\left[\left\|u_{\omega}-U\right\|_{X(0, l)}\left(\left\|u_{\omega}\right\|_{X(0, l)}^{2}+\|U\|_{X(0, l)}^{2}\right)\right. \\
\left.\quad+\left\|u_{\omega}-U\right\|_{X(0, l)}^{2}\left(\left\|u_{\omega}\right\|_{X(0, l)}+\|U\|_{X(0, l)}\right)\right] \\
\leq C l^{\alpha}\left(M^{2}+Q^{2}\right)\left[\left\|u_{\omega}-U\right\|_{X(0, l)}^{2}+\left\|u_{\omega}-U\right\|_{X(0, l)}\right] .
\end{gathered}
$$

Equations (19) and (18) can deduce that

$$
\begin{aligned}
&\left\|u_{\omega}-U\right\|_{L^{q}\left((0, l), W^{1, r}\left(\mathbb{R}^{N}\right)\right)} \\
& \leq C \varepsilon_{\omega}+C l^{\alpha}\left(M^{2}+Q^{2}\right) \\
& \times\left[\left\|u_{\omega}-U\right\|_{X(0, l)}^{2}+\left\|u_{\omega}-U\right\|_{X(0, l)}\right] .
\end{aligned}
$$

The conclusion (12) can be obtained from the above inequality, if we can show

$$
\left\|u_{\omega}-U\right\|_{X(0, l)} \underset{|\omega| \rightarrow \infty}{\longrightarrow} 0 .
$$

Divide $[0, l]$ into subintervals $\left[t_{i}, t_{i+1}\right], i=0, \ldots, J-1$, with $t_{0}=0, t_{J}=l$ such that in each subinterval, we have

$$
\begin{aligned}
C l^{\alpha}( & \left\|u_{\omega}\right\|_{X\left(t_{i}, t_{i+1}\right)}^{2}+\|U\|_{X\left(t_{i}, t_{i+1}\right)}^{2} \\
& \left.+\left\|u_{\omega}\right\|_{X\left(t_{i}, t_{i+1}\right)}+\|U\|_{X\left(t_{i}, t_{i+1}\right)}\right) \leq \frac{1}{2},
\end{aligned}
$$

where $J$ only depends on $M$ and $Q$.

In the initial interval $\left[t_{0}, t_{1}\right]$, since $u_{\omega}\left(t_{0}\right)=U\left(t_{0}\right)=\varphi$, (19), (18), and (22) deduce that

$$
\begin{aligned}
& \left\|u_{\omega}-U\right\|_{X\left(t_{0}, t_{1}\right)} \\
& \quad \leq C \varepsilon_{\omega}+\frac{1}{2}\left[\left\|u_{\omega}-U\right\|_{X\left(t_{0}, t_{1}\right)}^{2}+\left\|u_{\omega}-U\right\|_{X\left(t_{0}, t_{1}\right)}\right],
\end{aligned}
$$

where we let $L^{q}\left(\left(t_{0}, t_{1}\right), W^{1, r}\left(\mathbb{R}^{N}\right)\right)=X\left(t_{0}, t_{1}\right)$ by the special choice of $(q, r)$.

Then by the continuity argument, we have

$$
\left\|u_{\omega}-U\right\|_{L^{\gamma}\left(\left(t_{0}, t_{1}\right), L^{\rho}\left(\mathbb{R}^{N}\right)\right)}<4 C \varepsilon_{\omega} .
$$

Since $u_{\omega}$ and $U$ both belong to $C\left([0, l], H^{1}\right)$, we choose $(q, r)=$ $(\infty, 2)$ and obtain

$$
\begin{aligned}
& \left\|u_{\omega}\left(t_{1}\right)-U\left(t_{1}\right)\right\|_{H^{1}} \\
& \quad \leq C \varepsilon_{\omega}+\frac{1}{2}\left[\left\|u_{\omega}-U\right\|_{X\left(t_{0}, t_{1}\right)}^{2}+\left\|u_{\omega}-U\right\|_{X\left(t_{0}, t_{1}\right)}\right] \\
& \quad<4 C \varepsilon_{\omega} .
\end{aligned}
$$


In the interval $\left[t_{1}, t_{2}\right]$, Strichartz estimates and inequalities (18), (22), and (25) deduce that

$$
\begin{aligned}
\left\|u_{\omega}-U\right\|_{L^{q}\left(\left(t_{1}, t_{2}\right), W^{1, r}\left(\mathbb{R}^{N}\right)\right)} & \\
\leq & \left\|u_{\omega}\left(t_{1}\right)-U\left(t_{1}\right)\right\|_{H^{1}}+C \varepsilon_{\omega} \\
& +\frac{1}{2}\left[\left\|u_{\omega}-U\right\|_{X\left(t_{1}, t_{2}\right)}^{2}+\left\|u_{\omega}-U\right\|_{X\left(t_{1}, t_{2}\right)}\right] \\
\leq & 5 C \varepsilon_{\omega}+\frac{1}{2}\left[\left\|u_{\omega}-U\right\|_{X\left(t_{1}, t_{2}\right)}^{2}+\left\|u_{\omega}-U\right\|_{X\left(t_{1}, t_{2}\right)}\right] .
\end{aligned}
$$

Let $L^{q}\left(\left(t_{1}, t_{2}\right), W^{1, r}\left(\mathbb{R}^{N}\right)\right)=X\left(t_{0}, t_{1}\right)$ and apply the continuity argument; we have

$$
\left\|u_{\omega}-U\right\|_{X\left(t_{1}, t_{2}\right)}<16 C \varepsilon_{\omega} .
$$

Furthermore, let $(q, r)=(\infty, 2)$ again; we have

$$
\left\|u_{\omega}\left(t_{2}\right)-U\left(t_{2}\right)\right\|_{H^{1}}<16 C \varepsilon_{\omega} .
$$

Therefore, by induction argument, we obtain

$$
\begin{gathered}
\left\|u_{\omega}-U\right\|_{X\left(t_{i}, t_{i+1}\right)}<4^{i+1} C \varepsilon_{\omega}, \\
\left\|u_{\omega}\left(t_{i+1}\right)-U\left(t_{i+1}\right)\right\|_{H^{1}}<4^{i+1} C \varepsilon_{\omega},
\end{gathered}
$$

where $i=0, \ldots, J-1$. have

Finally, put all estimates in each subinterval together; we

$$
\left\|u_{\omega}-U\right\|_{X(0, l)} \leq \sum_{i=0}^{J-1} 4^{i+1} C \varepsilon_{\omega}<4^{J+1} C \varepsilon_{\omega} \underset{|\omega| \rightarrow \infty}{\longrightarrow} 0,
$$

which shows (21) is true and finishes the proof of lemma.

At the end of section, we give a blow-up alternative for (HS) (or (OHS)), which is useful for the proof of Theorem 3.

Lemma 9. For any initial data $\varphi \in H^{1}\left(\mathbb{R}^{N}\right)$ and $\gamma>2$, there exists a unique $H^{1}$ solution $U$ of $(H S)$ (or $(O H S)$ ) defined on the maximal life interval $\left[0, T_{\max }\right)$ with $0<T_{\max } \leq \infty$. If one supposes

$$
U \in L^{12 /(8-\gamma)}\left(\left(0, T_{\max }\right), L^{6 N /(3 N-2-2 \gamma)}\left(\mathbb{R}^{N}\right)\right),
$$

then one has $T_{\max }=\infty$ and $U \in L^{q}\left((0, \infty), W^{1, r}\left(\mathbb{R}^{N}\right)\right)$ with any admissible pair $(q, r)$.

Proof. We assume $T_{\max }<\infty$; then according to Proposition 1, we obtain $\|U\|_{X\left(0, T_{\max }\right)}=\infty$ and for any $T \in\left[0, T_{\max }\right)$, $\|U\|_{X(0, T)}<\infty$. Since $U \in L^{12 /(8-\gamma)}\left(\left(0, T_{\max }\right), L^{6 N /(3 N-2-2 \gamma)}\right.$ $\left(\mathbb{R}^{N}\right)$ ), we can choose $T_{0}$ sufficiently close to $T_{\max }$ such that

$$
\|U\|_{L^{12 /(8-\gamma)}\left(\left(T_{0}, T_{\max }\right), L^{6 N /(3 N-2-2 \gamma)}\left(\mathbb{R}^{N}\right)\right)}<\mathcal{E},
$$

where $\varepsilon$ is sufficiently small. that

For any admissible pair $(q, r)$, Strichartz estimate deduces

$$
\begin{aligned}
& \|\nabla U\|_{L^{q}\left(\left(T_{0}, T\right), L^{r}\left(\mathbb{R}^{N}\right)\right)} \\
& \leq\left\|\nabla U\left(T_{0}\right)\right\|_{L^{2}} \\
& \quad+\left\|\nabla\left[\left(|x|^{-\gamma} *|U|^{2}\right) U\right]\right\|_{L^{6 /(8-\gamma)}\left(\left(T_{0}, T\right), L^{6 N /(3 N-4+2 \gamma)}\left(\mathbb{R}^{N}\right)\right)} .
\end{aligned}
$$

Note that

$$
\begin{aligned}
\nabla & {\left[\left(|x|^{-\gamma} *|U|^{2}\right) U\right] } \\
& =\left[|x|^{-\gamma} * \nabla\left(|U|^{2}\right)\right] U+\left(|x|^{-\gamma} *|U|^{2}\right) \nabla U .
\end{aligned}
$$

It follows from Hardy-Littlewood-Sobolev inequality and Hölder inequality that

$$
\begin{aligned}
& \left\|\nabla\left[\left(|x|^{-\gamma} *|U|^{2}\right) U\right]\right\|_{L^{6 /(8-\gamma)}\left(\left(T_{0}, T\right), L^{6 N /(3 N-4+2 \gamma)}\left(\mathbb{R}^{N}\right)\right)} \\
& \quad \leq\|\nabla U\|_{L^{\infty}\left(\left(T_{0}, T\right), L^{2}\right)}\|U\|_{L^{12 /(8-\gamma)}\left(\left(T_{0}, T_{\max }\right), L^{6 N /(3 N-2-2 \gamma)}\left(\mathbb{R}^{N}\right)\right)}^{2} \\
& \quad \leq C \varepsilon^{2}\|U\|_{X\left(T_{0}, T\right)} .
\end{aligned}
$$

From (33) and (35), we obtain

$$
\|\nabla U\|_{L^{q}\left(\left(T_{0}, T\right), L^{r}\left(\mathbb{R}^{N}\right)\right)} \leq C\left\|\nabla U\left(T_{0}\right)\right\|_{L^{2}}+C \varepsilon^{2}\|U\|_{X\left(T_{0}, T\right)} .
$$

By Sobolev embedding and the definition of $X\left(T_{0}, T\right)$, we have

$$
\|U\|_{X\left(T_{0}, T\right)} \leq C\left\|U\left(T_{0}\right)\right\|_{H^{1}}+C \varepsilon^{2}\|U\|_{X\left(T_{0}, T\right)} .
$$

If we choose $C \varepsilon^{2}<1 / 2$, we have $\|U\|_{X\left(T_{0}, T\right)} \leq 2 C\left\|U\left(T_{0}\right)\right\|_{H^{1}}$, which is uniformly bounded for any $T$. Then let $T$ converge to $T_{\max }$; we have $\|U\|_{X\left(T_{0}, T_{\max }\right)} \leq 2 C\left\|U\left(T_{0}\right)\right\|_{H^{1}}$, which is a contradiction. Now, we know $U \in X(0, \infty)$. Then, by (33) and (35), we know $\nabla U \in L^{q}\left((0, \infty), L^{r}\left(\mathbb{R}^{N}\right)\right)$. The similar way can show $U \in L^{q}\left((0, \infty), L^{r}\left(\mathbb{R}^{N}\right)\right)$; thus we finish the proof.

\section{The Proof of Theorem 2}

In this section, we prove the Theorem 2. In view of Lemma 8, we only need to show that the solution $u_{\omega}$ of (OHS) exists in the interval $[0, T]$ for sufficiently large $\omega$ and the condition (10) holds.

Proof. For any $0<T<S_{\max }$, let $M:=2\|U(t)\|_{X(0, T)}$ and $\|\theta\|_{L^{\infty}} \leq A$; furthermore, we have $|I(\theta)| \leq A$, where the norm $X$ is defined as (11). Divide the interval $[0, T]$ into subintervals $\left[t_{i}, t_{i+1}\right], i=0, \ldots, J-1$, and $t_{0}=0, t_{J}=T$, such that in each subinterval $\left[t_{i}, t_{i+1}\right]$, we have

$$
\|U(t)\|_{X\left(t_{i}, t_{i+1}\right)} \leq \varepsilon,
$$

where $J$ only depends on $M$ and $T$, and $\varepsilon$ is a sufficiently small constant which is chosen later.

In each subinterval $\left[t_{i}, t_{i+1}\right]$, the integral forms (3), (19) (let $u_{\omega}=0, \theta(\omega s)=I(\theta)$ and $\left.[0, l]=\left[t_{i}, t_{i+1}\right]\right)$, and (38) apply

$$
\begin{aligned}
& \left\|e^{i\left(t-t_{i}\right) \Delta} U\left(t_{i}\right)\right\|_{X\left(t_{i}, t_{i+1}\right)} \\
& \quad \leq\|U(t)\|_{X\left(t_{i}, t_{i+1}\right)}+\mathrm{CAT}^{\alpha}\|U\|_{X\left(t_{i}, t_{i+1}\right)}^{3} \\
& \quad \leq \varepsilon+\mathrm{CAT}^{\alpha} \varepsilon^{3} \leq 2 \varepsilon,
\end{aligned}
$$

where we choose $\varepsilon$ sufficiently small such that $\mathrm{CAT}^{\alpha} \varepsilon^{2}<1$ and

$$
\alpha= \begin{cases}1, & \text { if } \gamma \leq 2 \\ 2-\frac{\gamma}{2}, & \text { if } 2<\gamma \leq 4\end{cases}
$$


On $\left[t_{0}, t_{1}\right]$, since $u_{\omega}\left(t_{0}\right)=U\left(t_{0}\right)=\varphi$, then by Strichartz estimate, (19) (let $U=0$ ), and (39), we obtain

$$
\begin{aligned}
\lim _{|\omega| \rightarrow \infty}\left\|u_{\omega}\right\|_{X\left(t_{0}, t_{1}\right)} & \\
\leq & \left\|e^{i t \Delta} \varphi\right\|_{X\left(t_{0}, t_{1}\right)} \\
& +\operatorname{CAT}^{\alpha}\left(\lim _{|\omega| \rightarrow \infty}\left\|u_{\omega}\right\|_{X\left(t_{0}, t_{1}\right)}\right)^{3} \\
\leq & 2 \varepsilon+\operatorname{CAT}^{\alpha}\left(\lim _{|\omega| \rightarrow \infty}\left\|u_{\omega}\right\|_{X\left(t_{0}, t_{1}\right)}\right)^{3} .
\end{aligned}
$$

Then the continuity argument deduces that

$$
\lim _{|\omega| \rightarrow \infty}\left\|u_{\omega}\right\|_{X\left(t_{0}, t_{1}\right)} \leq 2\left\|e^{i t \Delta} \varphi\right\|_{X\left(t_{0}, t_{1}\right)} \leq M,
$$

if $\varepsilon$ is sufficiently small such that $8 \mathrm{CAT}^{\alpha} \varepsilon<1$, where the second inequality comes from the Strichartz estimate and the definition of $M$.

Therefore, if $|\omega|$ is sufficiently large, the solution $u_{\omega}$ exists on $\left[t_{0}, t_{1}\right]$ and (10) holds. By Lemma 8 , we have $\lim _{|\omega| \rightarrow \infty}\left\|u_{\omega}\left(t_{1}\right)-U\left(t_{1}\right)\right\|_{H^{1}}=0$.

On $\left[t_{1}, t_{2}\right]$, Strichartz estimate and (19) deduce

$$
\begin{aligned}
& \lim _{|\omega| \rightarrow \infty}\left\|u_{\omega}\right\|_{X\left(t_{1}, t_{2}\right)} \\
& \leq \lim _{|\omega| \rightarrow \infty}\left\|e^{i\left(t-t_{1}\right) \Delta} u_{\omega}\left(t_{1}\right)\right\|_{X\left(t_{1}, t_{2}\right)} \\
& \quad+\operatorname{CAT}^{\alpha}\left(\lim _{|\omega| \rightarrow \infty}\left\|u_{\omega}\right\|_{X\left(t_{1}, t_{2}\right)}\right)^{3} \\
& \leq \lim _{|\omega| \rightarrow \infty}\left\|u_{\omega}\left(t_{1}\right)-U\left(t_{1}\right)\right\|_{H^{1}}+\left\|e^{i\left(t-t_{1}\right) \Delta} U\left(t_{1}\right)\right\|_{X\left(t_{1}, t_{2}\right)} \\
& \quad+\operatorname{CAT}^{\alpha}\left(\lim _{|\omega| \rightarrow \infty}\left\|u_{\omega}\right\|_{X\left(t_{1}, t_{2}\right)}\right)^{3} \\
& \leq\left\|e^{i\left(t-t_{1}\right) \Delta} U\left(t_{1}\right)\right\|_{X\left(t_{1}, t_{2}\right)}+\operatorname{CAT}^{\alpha}\left(\lim _{|\omega| \rightarrow \infty}\left\|u_{\omega}\right\|_{X\left(t_{1}, t_{2}\right)}\right)^{3} .
\end{aligned}
$$

Applying the continuity argument again, we have

$$
\lim _{|\omega| \rightarrow \infty}\left\|u_{\omega}\right\|_{X\left(t_{1}, t_{2}\right)} \leq 2\left\|e^{i\left(t-t_{1}\right) \Delta} U\left(t_{1}\right)\right\|_{X\left(t_{1}, t_{2}\right)} \leq M .
$$

Therefore, when $|\omega|$ is sufficiently large, the solution $u_{\omega}$ still exists in $\left[t_{1}, t_{2}\right]$, and (10) holds. Furthermore, by Lemma 8, we have $\lim _{|\omega| \rightarrow \infty}\left\|u_{\omega}\left(t_{2}\right)-U\left(t_{2}\right)\right\|_{H^{1}}=0$.

By induction, on each subinterval $\left[t_{i}, t_{i+1}\right]$, we always have $\lim _{|\omega| \rightarrow \infty}\left\|u_{\omega}\right\|_{X\left(t_{i}, t_{i+1}\right)} \leq M$ since the number of subintervals is finite, which only depends on $M$ and $T$. So, if $|\omega|$ is sufficiently large, the solution $u_{\omega}$ exists in $[0, T]$, and the condition (10) holds. Therefore, Theorem 2 follows from Lemma 8; thus we complete the proof.

\section{The Proof of Theorem 3}

The last section is devoted to the proof of Theorem 3. By blowup alternative in Proposition 1, the key point is to show the boundness of $\left\|u_{\omega}\right\|_{X(0, \infty)}$ as $\omega$ being sufficiently large.
Proof. The global existence of solution $U$ of (HS) is followed from Lemma 9. For any $T \in(0, \infty)$, Theorem 2 shows that the solution $u_{\omega}$ of (OHS) exists in $[0, T]$ for sufficiently large $\omega$ and $u_{\omega} \rightarrow U$ in $L^{q}\left((0, T), W^{1, r}\left(\mathbb{R}^{N}\right)\right)$ as $|\omega| \rightarrow \infty$ with any admissible pair $(q, r)$. In particular,

$$
\lim _{\omega \rightarrow \infty}\left\|u_{\omega}-U\right\|_{X(0, T)}=0 .
$$

Choose $T_{0}$ sufficiently large such that (32) holds, where $\varepsilon$ satisfies the smallness in the proof of Lemma 9. According to the proof of Lemma 9, we have

$$
\|U\|_{X\left(T_{0}, \infty\right)} \leq 2 C\left\|U\left(T_{0}\right)\right\|_{H^{1}} .
$$

For any $S \in\left[T_{0}, T_{\max }^{\omega}\right]$, triangle inequality deduces that

$$
\begin{aligned}
& \left\|u_{\omega}\right\|_{X\left(T_{0}, S\right)} \\
& \quad \leq\left\|u_{\omega}-U\right\|_{X\left(T_{0}, S\right)}+\|U\|_{X\left(T_{0}, S\right)} \\
& \quad \leq\left\|u_{\omega}-U\right\|_{X\left(T_{0}, S\right)}+2 C\left\|U\left(T_{0}\right)\right\|_{H^{1}} .
\end{aligned}
$$

Let $|\omega|$ go to $\infty$ on both sides; then we obtain from (45) (let $T=S$ ) that

$$
\lim _{\omega \rightarrow \infty}\left\|u_{\omega}\right\|_{X\left(T_{0}, S\right)} \leq 2 C\left\|U\left(T_{0}\right)\right\|_{H^{1}} .
$$

The arbitrary value of $S$ shows that when $\omega$ is sufficiently large, the solution $u_{\omega}$ is global existence by blow-up alternative in Proposition 1. Then Lemma 9 can deduce that

$$
\lim _{\omega \rightarrow \infty}\left\|u_{\omega}\right\|_{L^{q}\left((0, \infty), W^{1, r}\left(\mathbb{R}^{N}\right)\right)}<\infty .
$$

Finally, we show that $u_{\omega} \rightarrow U$ in $L^{q}\left((0, \infty), W^{1, r}\left(\mathbb{R}^{N}\right)\right)$ as $|\omega| \rightarrow \infty$ for all admissible pairs $(q, r)$. Theorem 2 shows that for any $T \in(0, \infty)$

$$
\lim _{\omega \rightarrow \infty}\left\|u_{\omega}-U\right\|_{L^{q}\left((0, T), W^{1, r}\left(\mathbb{R}^{N}\right)\right)}=0 ;
$$

therefore, our attention is focused on

$$
\lim _{\omega \rightarrow \infty}\left\|u_{\omega}-U\right\|_{L^{q}\left((T, \infty), W^{1, r}\left(\mathbb{R}^{N}\right)\right)}=0 .
$$

We note that

$$
\begin{aligned}
& u_{\omega}(T+t)-U(T+t) \\
& =e^{i \cdot \Delta}\left[u_{\omega}(T)-U(T)\right] \\
& +i \int_{0}^{t} \theta(\omega(T+s)) e^{i(t-s) \Delta} \\
& \quad \times\left[\left(|x|^{-\gamma} *\left|u_{\omega}\right|^{2}\right) u_{\omega}(T+s)\right. \\
& \left.\quad-\left(|x|^{-\gamma} *|U|^{2}\right) U(T+s)\right] d s \\
& +i \int_{0}^{t}[\theta(\omega(T+s))-I(\theta)] e^{i(t-s) \Delta} \\
& \quad \times\left(|x|^{-\gamma} *|U|^{2}\right) U(T+s) d s \\
& :=(I)+(I I)+(I I I) .
\end{aligned}
$$


Strichartz estimates and Theorem 2 show that

$$
\|(I)\|_{L^{q}\left((0, \infty), W^{1, r}\left(\mathbb{R}^{N}\right)\right)}:=\varepsilon_{\omega}^{1} \underset{|\omega| \rightarrow \infty}{\longrightarrow} 0 .
$$

By Lemma 9, we know $U \in L^{6 /(8-\gamma)}\left((0, \infty), W^{1,6 N /(2 N+2 \gamma-4)}\right)$; then Lemma 7 deduces that

$$
\|(I I I)\|_{L^{q}\left((0, \infty), W^{1, r}\left(\mathbb{R}^{N}\right)\right)}:=\varepsilon_{\omega}^{2} \underset{|\omega| \rightarrow \infty}{\longrightarrow} 0 .
$$

Since

$$
\begin{aligned}
& \left(|x|^{-\gamma} *\left|u_{\omega}\right|^{2}\right) u_{\omega}-\left(|x|^{-\gamma} *|U|^{2}\right) U \\
& \quad=\left(|x|^{-\gamma} *\left(\left|u_{\omega}\right|^{2}-|U|^{2}\right)\right) u_{\omega}+\left(|x|^{-\gamma} *|U|^{2}\right)\left(u_{\omega}-U\right),
\end{aligned}
$$

then it follows from Strichartz estimates, Hardy-LittlewoodSobolev inequality, and Hölder inequality that

$$
\begin{aligned}
&\|(I I)\|_{L^{q}\left((0, \infty), W^{1, r}\left(\mathbb{R}^{N}\right)\right)} \\
& \leq C\|U\|_{L^{12 /(8-\gamma)}\left((T, \infty), L^{6 N /(3 N-2-2 \gamma)}\left(\mathbb{R}^{N}\right)\right)}^{2} \\
& \times\left\|u_{\omega}-U\right\|_{L^{\infty}\left((T, \infty), H^{1}\left(\mathbb{R}^{N}\right)\right)} \\
&+C\|U\|_{L^{\infty}\left((T, \infty), H^{1}\left(\mathbb{R}^{N}\right)\right)} \\
& \times\|U\|_{L^{12 /(8-\gamma)}\left((T, \infty), L^{6 N /(3 N-2-2 \gamma)}\left(\mathbb{R}^{N}\right)\right)} \\
& \times\left\|u_{\omega}-U\right\|_{L^{12 /(8-\gamma)}\left((T, \infty), L^{6 N /(3 N-2-2 \gamma)}\left(\mathbb{R}^{N}\right)\right)} \\
&+C\left\|u_{\omega}-U\right\|_{L^{12 /(8-\gamma)}\left((T, \infty), L^{6 N /(3 N-2-2 \gamma)}\left(\mathbb{R}^{N}\right)\right)}^{2} \\
& \times\|U\|_{L^{\infty}\left((T, \infty), H^{1}\left(\mathbb{R}^{N}\right)\right)} \\
&+\left\|u_{\omega}-U\right\|_{L^{\infty}\left((T, \infty), H^{1}\left(\mathbb{R}^{N}\right)\right)} \\
& \times\left\|u_{\omega}-U\right\|_{L^{12 /(8-\gamma)}\left((T, \infty), L^{6 N /(3 N-2-2 \gamma)}\left(\mathbb{R}^{N}\right)\right)} \\
& \times\left\|u_{\omega}\right\|_{L^{12 /(8-\gamma)}\left((T, \infty), L^{6 N /(3 N-2-2 \gamma)}\left(\mathbb{R}^{N}\right)\right)}
\end{aligned}
$$

By Sobolev embedding $\dot{H}^{1,6 N /(3 N+4-2 \gamma)} \hookrightarrow L^{6 N /(3 N-2-2 \gamma)}$ and interpolation, for any time interval $I$, we have

$$
\begin{aligned}
& \|\psi\|_{L^{12 /(8-\gamma)}\left(I, L^{6 N /(3 N-2-2 \gamma)}\right)} \\
& \quad \leq C\|\nabla \psi\|_{L^{12 /(8-\gamma)}\left(I, L^{6 N /(3 N+4-2 \gamma)}\right)} \\
& \quad \leq C\|\nabla \psi\|_{L^{12 /(8-\gamma)}\left(I, L^{6 N /(3 N-8+\gamma)}\right)}^{(4-2 \gamma /(\gamma-)}\|\nabla \psi\|_{L^{\infty}\left(I, L^{2}\right)}^{(3 \gamma-12) /(\gamma-8)} .
\end{aligned}
$$

We set $Z(I)=L^{\infty}\left(I, H^{1}\left(\mathbb{R}^{N}\right)\right) \cap L^{12 /(8-\gamma)}\left(I, W^{1,6 N /(3 N-8+\gamma)}\right.$ $\left(\mathbb{R}^{N}\right)$. By (52)-(57), we have

$$
\begin{aligned}
\left\|u_{\omega}(t)-U(t)\right\|_{L^{q}\left((T, \infty), W^{1, r}\left(\mathbb{R}^{N}\right)\right.} & \\
\leq & \varepsilon_{\omega}^{1}+\varepsilon_{\omega}^{2} \\
& +C\|U\|_{L^{12 /(8-\gamma)}\left((T, \infty), L^{6 N /(3 N-2-2 \gamma)}\left(\mathbb{R}^{N}\right)\right)}^{2}\left\|u_{\omega}-U\right\|_{Z(T, \infty)} \\
& +C M\|U\|_{L^{12 /(8-\gamma)}\left((T, \infty), L^{6 N /(3 N-2-2 \gamma)}\left(\mathbb{R}^{N}\right)\right)}\left\|u_{\omega}-U\right\|_{Z(T, \infty)}
\end{aligned}
$$

$$
\begin{aligned}
& +C M\left\|u_{\omega}-U\right\|_{Z(T, \infty)}^{2}+C\left\|u_{\omega}-U\right\|_{Z(T, \infty)}^{3} \\
& +C\|U\|_{L^{12 /(8-\gamma)}\left((T, \infty), L^{6 N /(3 N-2-2 \gamma)}\left(\mathbb{R}^{N}\right)\right)}\left\|u_{\omega}-U\right\|_{Z(T, \infty)}^{2},
\end{aligned}
$$

where we suppose $\|U\|_{X(0, \infty)} \leq M$.

Choose $T$ sufficiently large such that

$$
\begin{aligned}
& C\|U\|_{L^{12 /(8-\gamma)}\left((T, \infty), L^{6 N /(3 N-2-2 \gamma)}\left(\mathbb{R}^{N}\right)\right)}^{2} \\
& +C M\|U\|_{L^{12 /(8-\gamma)}\left((T, \infty), L^{6 N /(3 N-2-2 \gamma)}\left(\mathbb{R}^{N}\right)\right)} \leq \frac{1}{2}, \\
& C\|U\|_{L^{12 /(8-\gamma)\left((T, \infty), L^{6 N /(3 N-2-2 \gamma)}\left(\mathbb{R}^{N}\right)\right)}} \leq 1 .
\end{aligned}
$$

Since $\left(L^{12 /(8-\gamma)}, L^{6 N /(3 N-8+\gamma)}\right)$ and $(\infty, 2)$ both are admissible pairs, then it follows from $(58)$ with $L^{q}\left((T, \infty), W^{1, r}\left(\mathbb{R}^{N}\right)\right)=$ $Z(T, \infty)$ that

$$
\begin{aligned}
\left\|u_{\omega}(t)-U(t)\right\|_{Z(T, \infty)} \\
\leq 2 \varepsilon_{\omega}^{1}+2 \varepsilon_{\omega}^{2}+2 C M\left\|u_{\omega}(t)-U(t)\right\|_{Z(T, \infty)}^{2} \\
\quad+2 C\left\|u_{\omega}(t)-U(t)\right\|_{Z(T, \infty)}^{3} \\
\quad+2\left\|u_{\omega}(t)-U(t)\right\|_{Z(T, \infty)}^{2} .
\end{aligned}
$$

The standard continuity argument shows that if $2 \varepsilon_{\omega}^{1}+2 \varepsilon_{\omega}^{2}$ is sufficiently small, that is, $\omega$ large enough, we have

$$
\left\|u_{\omega}(t)-U(t)\right\|_{Z(T, \infty)} \leq 4 \varepsilon_{\omega}^{1}+4 \varepsilon_{\omega}^{2} \underset{|\omega| \rightarrow \infty}{\longrightarrow} 0 .
$$

Finally, (53), (54), (58), and (61) deduce that

$$
\lim _{\omega \rightarrow \infty}\left\|u_{\omega}-U\right\|_{L^{q}\left((T, \infty), W^{1, r}\left(\mathbb{R}^{N}\right)\right)}=0 .
$$

\section{Conflict of Interests}

The author declares that there is no conflict of interests regarding the publication of this paper.

\section{Acknowledgments}

The author thanks the anonymous referees for constructive remarks.

\section{References}

[1] J. Fröhlich and E. Lenzmann, "Mean-field limit of quantum Bose gases and nonlinear Hartree equation," in Séminaire: Équations aux Dérivées Partielles (2003-2004), Exp. No. XIX, p. 26, École Polytechnique, Palaiseau, France, 2004.

[2] Y. B. Gaididei, K. Ø. Rasmussen, and P. L. Christiansen, "Nonlinear excitations in two-dimensional molecular structures with impurities," Physical Review E, vol. 52, no. 3, pp. 2951-2962, 1995.

[3] L. P. Pitaevskii, "Vortex lines in an imperfect Bose gas," Soviet Physics-Journal of Experimental and Theoretical Physics, vol. 13, pp. 451-454, 1961. 
[4] T. Cazenave, Semilinear Schrödinger Equations, vol. 10 of Courant Lecture Notes in Mathematics, New York University, Courant Institute of Mathematical Sciences, New York, NY, USA; American Mathematical Society, Providence, RI, USA, 2003.

[5] T. Cazenave and F. B. Weissler, "The Cauchy problem for the nonlinear Schrödinger equation in $H^{1}$," Manuscripta Mathematica, vol. 61, no. 4, pp. 477-494, 1988.

[6] C. X. Miao, G. X. Xu, and L. F. Zhao, "Global well-posedness and scattering for the energy-critical, defocusing Hartree equation for radial data," Journal of Functional Analysis, vol. 253, no. 2, pp. 605-627, 2007.

[7] C. X. Miao, G. X. Xu, and L. F. Zhao, "Global well-posedness, scattering and blow-up for the energy-critical, focusing Hartree equation in the radial case," Colloquium Mathematicum, vol.114, no. 2, pp. 213-236, 2009.

[8] C. X. Miao, G. X. Xu, and L. F. Zhao, "The Cauchy problem of the Hartree equation," Journal of Partial Differential Equations, vol. 21, no. 1, pp. 22-44, 2008.

[9] T. Cazenave and M. Scialom, "A Schrödinger equation with time-oscillating nonlinearity," Revista Matemática Complutense, vol. 23, no. 2, pp. 321-339, 2010.

[10] D. Fang and Z. Han, "A Schrödinger equation with timeoscillating critical nonlinearity," Nonlinear Analysis: Theory, Methods \& Applications A: Theory and Methods, vol. 74, no. 14, pp. 4698-4708, 2011.

[11] R. S. Strichartz, "Restrictions of Fourier transforms to quadratic surfaces and decay of solutions of wave equations," Duke Mathematical Journal, vol. 44, no. 3, pp. 705-714, 1977.

[12] J. Ginibre and G. Velo, "The global Cauchy problem for the nonlinear Schrödinger equation revisited," Annales de l'Institut Henri Poincaré. Analyse Non Linéaire, vol. 2, no. 4, pp. 309-327, 1985.

[13] K. Yajima, "Existence of solutions for Schrödinger evolution equations," Communications in Mathematical Physics, vol. 110, no. 3, pp. 415-426, 1987.

[14] M. Keel and T. Tao, "Endpoint Strichartz estimates," The American Journal of Mathematics, vol. 120, no. 5, pp. 955-980, 1998.

[15] T. Kato, Perturbation Theory for Linear Operators, Springer, Berlin, Germany, 2nd edition, 1980. 


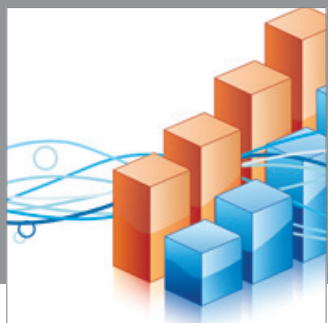

Advances in

Operations Research

mansans

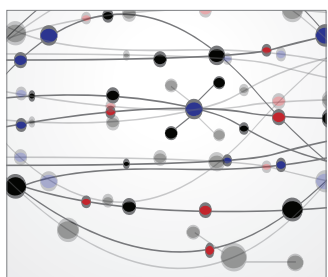

The Scientific World Journal
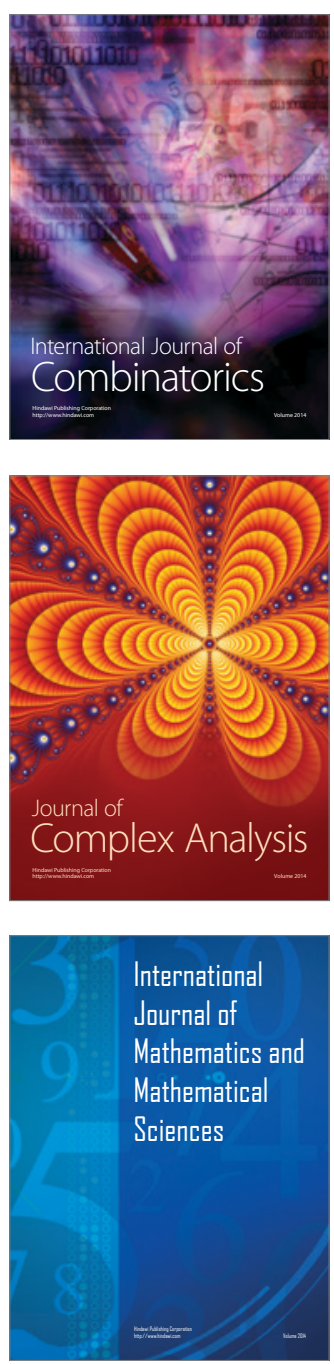
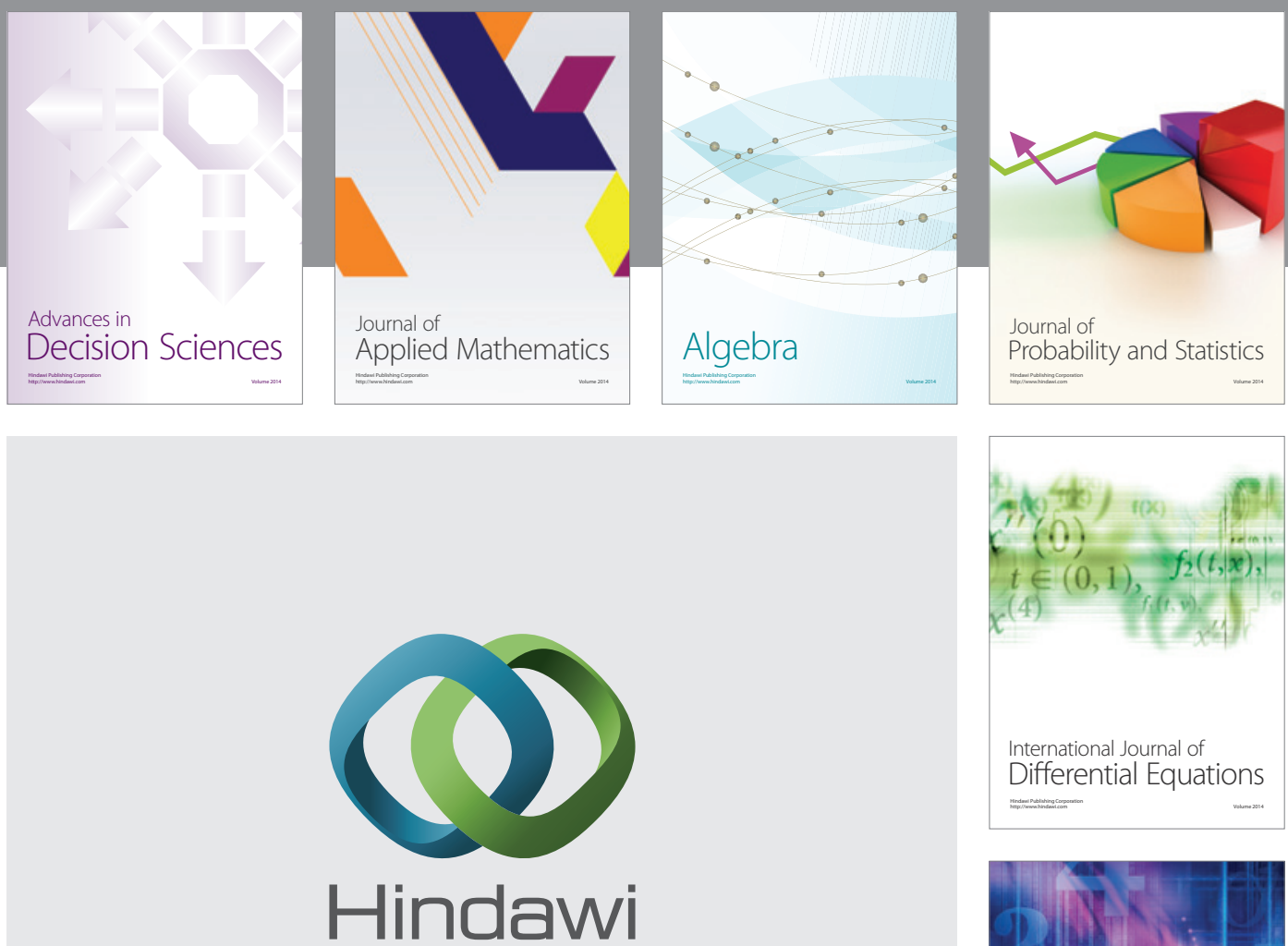

Submit your manuscripts at http://www.hindawi.com
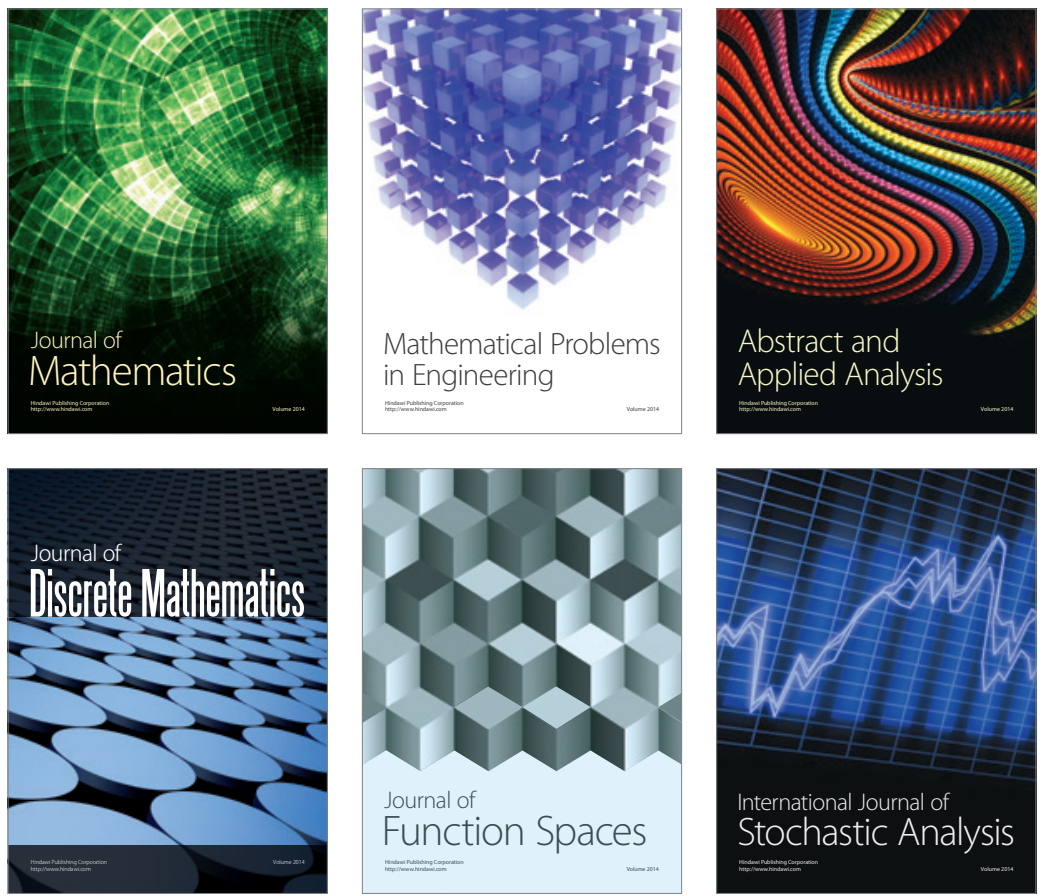

Journal of

Function Spaces

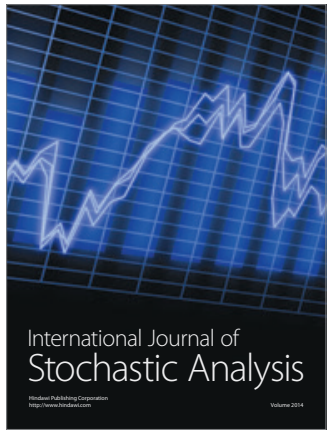

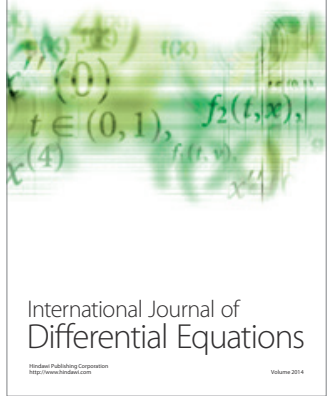
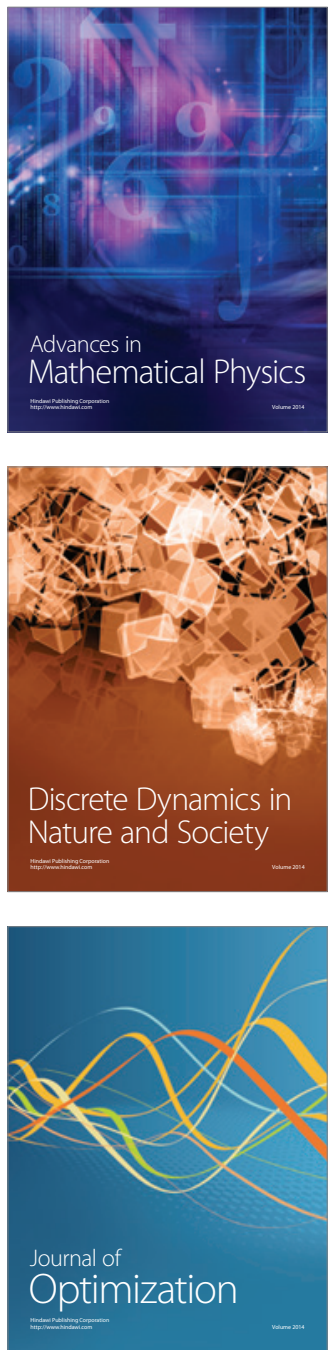